\title{
Variabilidad en el crecimiento de plántulas de ocho procedencias de Abies religiosa (H.B.K.) Schlecht. et Cham., en condiciones de vivero
}

\section{Growth variability in seedlings of eight provenances of Abies religiosa (H.B.K.) Schlecht. et Cham., in nursery conditions}

\author{
Héctor Mario Benavides-Meza1, Maira Oriana Gazca Guzmán², \\ Stephanie Fabiola López López², Francisco Camacho Morfín1, \\ Diana Young Fernández Grandizo², María del Pilar de la Garza \\ López de Lara ${ }^{3}$ y Felipe Nepamuceno Martínez 3
}

\begin{abstract}
RESUMEN
Abies religiosa presenta una amplia distribución en la República Mexicana pues se extiende desde Sinaloa y San Luis Potosí hasta Puebla y Tlaxcala e incluso se encuentran poblaciones de esta especie en Guatemala. En el Valle de México se ubica en las zonas montañosas que rodean al mismo y sus bosques tienen una gran importancia ecológica y social, pues estas masas arboladas forman parte del bosque periurbano del Área Metropolitana de la Ciudad de México. No obstante lo anterior, estos sitios arbolados están sujetos a un grave deterioro por lo que es necesario buscar alternativas para su restauración. El objetivo de este trabajo fue evaluar la variación en el crecimiento de plántulas de ocho procedencias bajo condiciones de vivero, para lo cual se utilizó un diseño experimental de ocho bloques al azar con 64 repeticiones. Los parámetros que se evaluaron fueron diámetro en la base del tallo y altura, en mediciones que se efectuaron trimestralmente durante un período de 10 meses. Las plántulas de la procedencia San Nicolás Coatepec presentaron los mayores valores en altura $(37,2 \mathrm{~cm})$, empero su tasa de crecimiento sólo difirió significativamente de la procedencia de Cofre de Perote. En cuanto a la tasa de crecimiento en diámetro, Coatepec (DISEFO) fue significativamente mayor al resto de las otras procedencias; sin embargo, en general no se observó ningún patrón de crecimiento relacionado con la distribución geográfica de las procedencias.
\end{abstract}

PALABRAS CLAVE:

Abies religiosa, crecimiento en vivero, Desierto de los Leones, procedencias, Valle de México, variabilidad.

\begin{abstract}
Abies religiosa shows a wide distribution in Mexico and extends from the States of Sinaloa and San Luis Potosí in the north part of Mexico up to Puebla and Tlaxcala in the south, but there are also populations of this species in Guatemala. The forests in the Valle de México region are located in the mountainous areas around Mexico City and socially and ecologically are very important because they
\end{abstract}


are a significant component of the periurban forest of this Metropolitan Area. Unfortunately these stands have been declining for the last 30 or more years and it is imperative to look for solutions to restore these areas. The objective for this project was to determine the variation in growth (height and basal diameter) among eight provenances of this species and was used a random block design with 64 replicates. Measurements were done every three months through a period of a year. Seedlings from San Nicolás Coatepec provenance showed the top values in height $(37,2 \mathrm{~cm})$, however the growth rate was only significantly different with Cofre de Perote. The biggest basal diameter growth was recorded in seedlings from Coatepec (DISEFO) provenance, and it was significantly different from the rest of provenances, but no pattern of geographicallydetermined variation was observed among the growth response from the different provenances.

\section{KEY WORDS:}

Abies religiosa, nursery growth, Desierto de los Leones, provenances, Valle de Mexico, variability

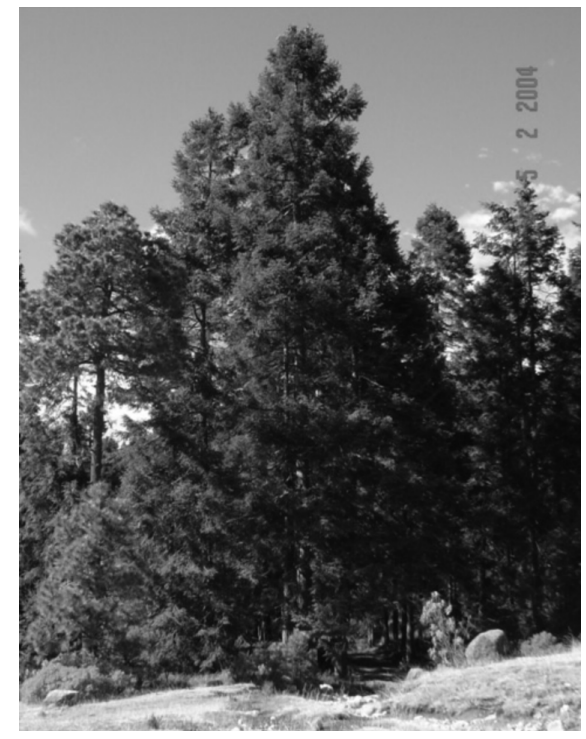

\section{INTRODUCCIÓN}

A la especie Abies religiosa (H.B.K.) Schlecht et Cham. se le conoce como pinabete, abeto, jalocote, jalocotl, bansu (otomí), acxoyatl (nahuatl) y oyamel, el cual es el más usado (Manzanilla-Bolio, 1974; Gómez-González, 2003). Es un árbol perennifolio que alcanza $40 \mathrm{~m}$ y en ocasiones $60 \mathrm{~m}$ de altura y presenta ramas extendidas o ligeramente ascendentes y verticiladas que forman una copa cónica. Presenta hojas alternas, dispuestas en espirales, lineares, torcidas en la base, con el ápice agudo y córneo, de color verde oscuro brillante en el haz y muy glaucas en el envés, de (20-30) mm, rara vez hasta 35 $\mathrm{mm}$ de largo y de $1,5 \mathrm{~mm}$ de ancho. Conos solitarios, erguidos, cilíndricos y oblongos, romos, resinosos, casi sésiles; de color violáceo a moreno violáceo y miden de (1016) $\mathrm{cm}$ de largo por (4-6) $\mathrm{cm}$ de ancho (Martínez, 1948; Calderón de Rzedowski y Rzedowski, 2001) (Fig. 1). Las semillas son

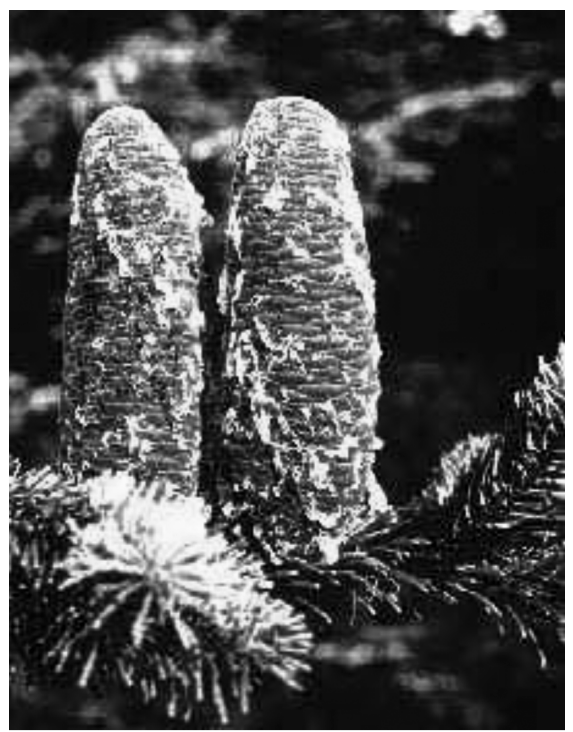

Figura 1. Árbol y conos de Abies religiosa.

FuENTES: (a) Héctor M. Benavides Meza, (b):http://www.conafor.gob.mx/portal/docs/secciones/reforestacion/ Fichas\%20Tecnicas/ Abies\%20religiosa.pdf 
cuneado-oblongas, de unos $9 \mathrm{~mm}$ a $10 \mathrm{~mm}$ de largo, aladas, lisas y de color castaño brillante (Martínez, 1953).

\section{Distribución y características de la especie}

El oyamel se distribuye en las partes altas montañosas desde Sinaloa y San Luis Potosí hasta Guatemala, entre los 2500 y 3660 msnm (Calderón de Rzedowski y Rzedowski, 2001). En México se encuentra principalmente a lo largo del Eje Neovolcánico entre los $17^{\circ} 30^{\prime}$ y $21^{\circ}$ $00^{\prime}$ latitud $\mathrm{N}$ y los $97^{\circ}$ a $104^{\circ} 00^{\prime}$ longitud $\mathrm{O}$, desde Veracruz hasta Michoacán y Jalisco (Martínez, 1953; Manzanilla-Bolio, 1974). Los bosques de $A$. religiosa se desarrollan en climas templados con precipitaciones de $1000 \mathrm{~mm}$ anuales; en suelos profundos, bien drenados; húmedos, ricos en materia orgánica y de neutros a ligeramente ácidos (MadrigalSánchez, 1967; Manzanilla-Bolio, 1974).

Los rodales de oyamel se encuentran puros en pequeños manchones o mezclados principalmente con Pinus montezumae Lamb., $P$. hartwegii Lindl., $P$. ayacahuite Ehren. ex Schlecht., $P$. douglasiana Mart., Pseudotsuga sp., Cupressus lindleyi Klotzsch ex Endl., Quercus sp., Alnus sp., Salix sp., Arctostahyllos sp. y Arbutus sp. (GómezGonzález, 2003).

\section{Abies religiosa en la Cuenca del Valle de México}

En la cuenca se encuentra desde El Chico hasta Epazoyucan y de Villa Nicolás Romero hasta Tlalpan y Milpa Alta; asimismo se distribuye de Texcoco a Amecameca, Juchitepec y Tepeapulco, desde los $2600 \mathrm{~m}$ en donde se encuentran escasos individuos en las barrancas y cañadas protegidas, hasta una altitud máxima de 3500 msnm. Las masas más densas se encuentran entre $2900 \mathrm{~m}$ y $3200 \mathrm{~m}$, en asociación frecuentemente con $P$. montezumae en el límite altitudinal inferior, $P$. psedostrobus Lindl., $P$. rudis Endl. y $C$. lindleyi en la parte media y $P$. hartwegii Lindl. en la parte superior (Madrigal-Sánchez, 1967; Calderón de Rzedowski y Rzedowski, 2001).

\section{Importancia ecológica y económica}

Los bosques de $A$. religiosa constituyen comunidades clímax que dominan las partes altas del Valle de México, cuyas características de cobertura vegetal y propiedades físicas del suelo permiten una eficiente absorción y retención del agua de lluvia; además de tener interés estético-recreativo y ser de especial importancia para la protección de fauna silvestre en los parques nacionales, cuatro de los cuales se encuentran en la Cuenca de México (Cumbres del Ajusco, Desierto de los Leones, Insurgente Miguel Hidalgo y Costilla y El Chico) y que suman 6556 ha (Madrigal-Sánchez, 1967; Nieto de Pascual-Pola, 1995). En función de lo anterior, estas masas arboladas son un importante componente de lo que se refiere a bosque periurbano (BenavidesMeza et al., 1994), que en este caso, al encontrarse en la periferia del Área Metropolitana de la Ciudad de México, adquiere especial relevancia por los servicios ambientales que genera para sus habitantes y el mejoramiento ecológico de la misma.

Aunado a lo anterior, la madera de esta especie es muy útil para la obtención de pulpa para papel debido al tamaño de su fibra y carencia de resina. De igual forma se emplea en la fabricación de cajas, canastas, empaques para alimentos, postes, vigas, morillos, pilotes, fajillas, así como leña y carbón. También es utilizado como árbol de navidad y se 
elaboran adornos con sus ramas (Madrigal-Sánchez, 1967; ManzanillaBolio, 1974).

\section{Declinación de Abies religiosa en el Desierto de los Leones}

A finales de la década de los setenta y principios de los ochenta se presentó en los bosques de $A$. religiosa y $P$. hartwegii del Desierto de los Leones, un proceso de declinación similar al reportado por botánicos europeos, el cual se manifestaba como una decoloración del follaje, disminución de la biomasa radical y aérea, caída prematura de acículas adultas en las coníferas, disminución tanto del crecimiento anual como de brotes y renuevos, así como un aumento en la susceptibilidad de los individuos arbóreos al ataque de insectos y enfermedades, que culminaba generalmente en una "muerte descendente" de los árboles, ya fueran dominantes o dominados en la masa (Alvarado et al., 1992; Alvarado-Rosales y Hernández-Tejeda, 2002; Cibrián, 1989; Cielsa y Macías, 1987). Se consideró desde entonces que la declinación comenzaba cuando los árboles eran alterados inicialmente por uno o más factores estresantes, los cuales deterioraban la salud y vigor del arbolado hasta el punto en que varios patógenos secundarios podían matar al huésped (Vázquez, 1987; Weaver y Stipes, 1988; GranadosSánchez y López-Ríos, 2001). Los datos disponibles hasta la fecha, señalan a la contaminación atmosférica (principalmente ozono) como el principal agente causal de la declinación forestal en dichos bosques (Granados-Sánchez y López-Ríos, 2001; González-Medina, et al., 2010). La situación de ésta área natural es preocupante ya que constituye una zona de depósito atmosférico elevado por estar ubicado en el sotavento de la cuenca y es el paso obligado del aire contaminado de la Zona Metropoli- tana de la Ciudad de México. De igual manera, la lluvia ácida es evidencia adicional de que la contaminación atmosférica afecta los ecosistemas forestales del Desierto de los Leones y la calidad del agua de lluvia que captan (Pérez-Suárez et al., 2006); pues los resultados de Saavedra-Romero et al. (2003), los cuales fueron obtenidos durante 1998, mostraban que en el desierto se presentaban Iluvias ácidas ( $\mathrm{pH}<5,65)$, especialmente en los meses con mayor precipitación promedio (agosto y octubre).

Derivado de esta situación, las dependencias operativas tienen la necesidad de realizar reforestaciones con especies nativas para acelerar procesos de sucesión vegetal que permitan repoblar en menor tiempo las áreas descubiertas y apoyar estas acciones con actividades de conservación de suelos (CONANP, 2006).

\section{Restauración de la cubierta arbórea en el área}

La restauración se define como el acto de regresar al estado original una comunidad, pero de acuerdo con Ashby (1987), la originalidad de un ecosistema es cuestionable si partimos de lo que se entienda por ecosistema original; así como el hecho de si el proceso de restauración pretende llevar la comunidad al estado antes de la degradación o a una etapa de sucesión más avanzada y el último de estos argumentos versa sobre la casi imposibilidad de alcanzar un estado único, lo cual llevaría a una condición estática que no es natural en un ecosistema. Debido a lo anterior, la meta de la restauración no es obtener una condición idéntica a la del ecosistema forestal considerado como original, sino a una cercana o parecida a la misma en la que se pretende regresar a un estado más saludable o vigoroso (Ashby, 1987; Rodríguez-Trejo, 2006). 
En relación con lo anterior, la utilización de procedencias de la especie que resultó afectada en el sitio puede ser una alternativa adecuada de restauración, con base en la variación genética de las especies forestales de amplia distribución, ligada a diferencias ambientales dentro de la misma (García-Quintana et al., 2007). La variación entre procedencias puede permitir el desarrollo de la especie en el sitio donde se encontraba la población original que fue afectada, como en el caso del Desierto de los Leones y con tal fin, los ensayos de procedencias son una herramienta útil para evaluar y valorar la respuesta de las mismas.

El presente trabajo forma parte de un gran proyecto que tuvo como objetivo central el proponer una alternativa sustentada en el uso de procedencias de Abies religiosa para la restauración de la cubierta arbórea en las áreas cementerio que presentan un severo deterioro en el Desierto de los Leones (Fig. 2). Por esta razón, la evaluación de las procedencias se inició desde su respuesta inicial a partir de su desarrollo en vivero y en la búsqueda, como lo plantean Climent et al. (2008), de plantas forestales de calidad genética adecuada para desarrollarse en las condiciones prevalecientes del Desierto de los Leones.

\section{Variación de Abies religiosa}

Las especies forestales con grandes áreas de distribución muestran, en general, una gran variación clinal controlada genéticamente. Ésta se ve reflejada en la plasticidad fenotípica de las características fisiológicas y morfológicas, como aquellas que están relacionadas con la

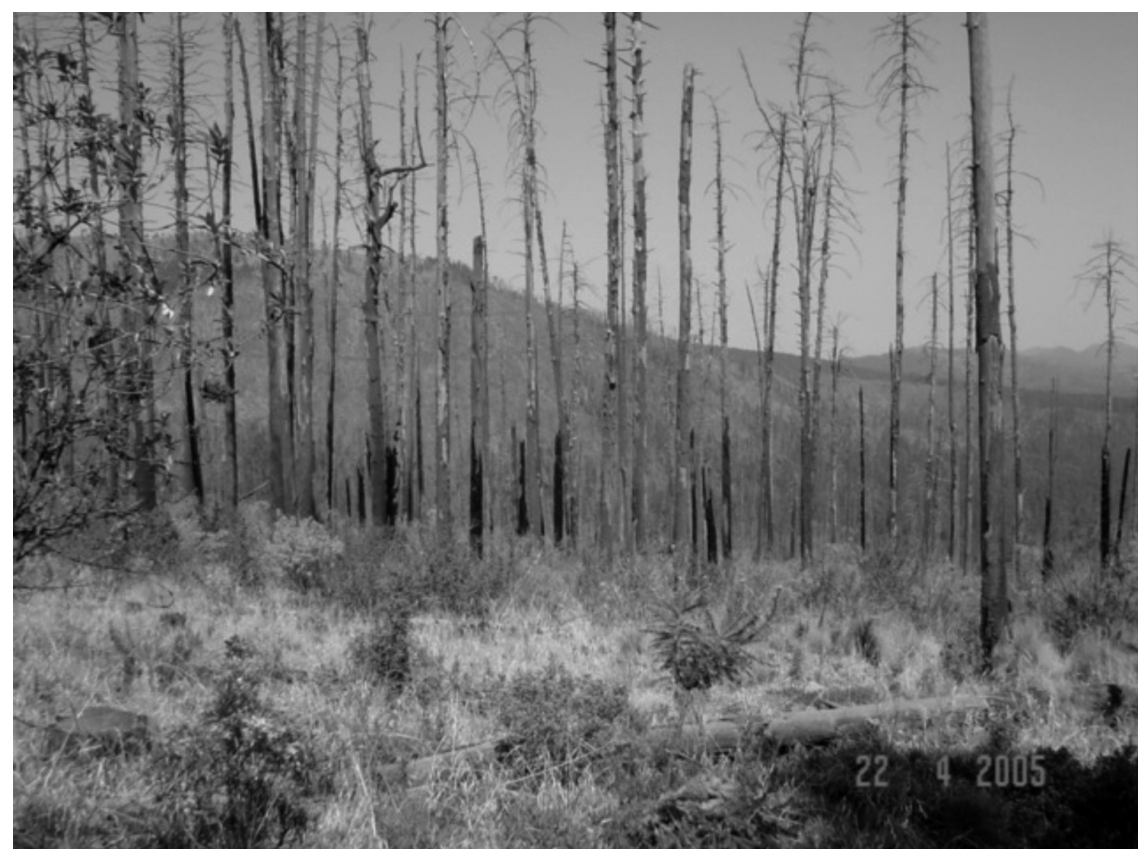

Figura 2. Área con arbolado muerto en pie de Abies religiosa en el Desierto de los Leones, D.F. 
resistencia por la adaptación a factores limitantes. La variación intraespecífica, es decir, la variación entre ecotipos de la misma especie se debe a la selección natural influida por los gradientes geográficos y ambientales que se presentan a lo largo del área de distribución y al aislamiento por discontinuidades que presentan las poblaciones (Zobel y Talbert, 1984). En la medida que una especie tiene un amplio rango de distribución, es posible encontrarla en diferentes altitudes, exposiciones, tipos de suelo $u$ otros factores ambientales y por ende sus poblaciones (procedencias) tenderán a contar con una variación genética y estar más diferenciadas. Ejemplo de lo anterior es frecuente encontrarlo en especies forestales de distribución geográfica amplia, las cuales pueden presentar una considerable variación en caracteres anatómicos, morfológicos y fisiológicos (Burley, 1969; Rodríguez-Trejo, 2006).

En el caso del oyamel se han realizado estudios sobre la variación morfológica y fisiológica en las semillas provenientes de diferentes localidades del Valle de México y Michoacán, entre los que destacan el realizado por la FAO (1971), donde se midió el porcentaje de germinación y numero de semillas por kilogramo. Nieto de Pascual-Pola et al. (2003) evaluaron la relación entre el tamaño y peso de los conos con la calidad de la semilla y reportan que su viabilidad es independiente del tamaño del cono. En forma similar, Cortés-Barrera et al. (2005) cuantificaron la viabilidad de las semillas y el potencial de regeneración de esta especie en dos localidades del Distrito Federal, de las cuales la proveniente de la Sierra del Ajusco presentó una mayor producción de semillas y una mayor capacidad germinativa a diferencia de la colectada en la Cañada de Contreras. Cabe destacar que dichos estudios se llevaron a cabo con el fin de tener un mayor conocimiento de la propagación de la especie como alternativa para su conservación.

Existen algunos estudios sobre aspectos dasométricos y de regeneración de los bosques de $A$. religiosa, incluso una evaluación en vivero con diferentes sustratos y tamaño de contenedor realizado por Santiago-Trinidad (2002), así como un diagnóstico de necesidades de fertilización por Moreno et al. (2002). No obstante lo anterior, hasta la fecha no se ha publicado ningún estudio relacionado con la variación en el crecimiento de plántulas de diferentes procedencias de oyamel, por lo que con base en la idea central del proyecto, se planteó el objetivo para el presente trabajo.

\section{OBJETIVO}

Determinar la variación en el crecimiento en diámetro y altura en plántulas provenientes de ocho procedencias de $A$. religiosa bajo condiciones de vivero.

\section{METODOLOGÍA}

\section{Recolección de semillas}

Se obtuvieron semillas de ocho localidades de $A$. religiosa procedentes tanto de los extremos de su distribución transversal en el Eje Neovolcánico, como de diferentes sitios ubicados en la zona central de la misma (Tabla 1). Los lotes 1 al 3 fueron colectados en los meses de noviembre y diciembre del 2003 por el grupo de investigadores y colaboradores del INIFAP/CENIDCOMEF. En cada sitio se cosecharon conos de 10 árboles maduros y sanos, ubicados a una distancia de $50 \mathrm{~m}$ o más uno del otro.; sin embargo, la semilla de estas procedencias no se mantuvo separada por árbol y se conformó un gran lote, al igual que el resto de las procedencias. El lote 4 fue donado por un distribuidor comercial 
Tabla 1. Sitios de colecta de las semillas de Abies religiosa.

\begin{tabular}{|c|c|c|c|c|}
\hline Núm.. & Procedencias & Clave & Coordenadas & $\begin{array}{l}\text { Altitud } \\
\text { (msnm) }\end{array}$ \\
\hline \multirow[t]{2}{*}{1} & Nevado de Colima, Jal. & NEC & $19^{\circ} 35^{\prime} 54^{\prime \prime} \mathrm{N}$ & \\
\hline & & & $103^{\circ} 35^{\prime} 05^{\prime \prime O}$ & 3197 \\
\hline \multirow[t]{2}{*}{2} & Cofre de Perote, Ver. & COP & $19^{\circ} 31^{\prime} 12^{\prime \prime} \mathrm{N}$ & \\
\hline & & & $97^{\circ} 09^{\prime} 30^{\prime \prime} \mathrm{O}$ & 3486 \\
\hline \multirow[t]{2}{*}{3} & Nevado de Toluca, Méx. & TOL & $19^{\circ} 11^{\prime} 03^{\prime \prime} \mathrm{N}$ & \\
\hline & & & $99^{\circ} 45^{\prime} 33^{\prime \prime} \mathrm{O}$ & 3229 \\
\hline \multirow[t]{2}{*}{4} & Coatepec, Méx. & EFO) $\mathrm{COH}$ & $19^{\circ} 00^{\prime} 00^{\prime \prime} \mathrm{N}$ & \\
\hline & & & $99^{\circ} 48^{\prime} 00^{\prime \prime O}$ & 2260 \\
\hline \multirow[t]{2}{*}{5} & San Nicolás Coatepec, Méx. & SNC & $19^{\circ} 06^{\prime} 58^{\prime \prime} \mathrm{N}$ & \\
\hline & & & $99^{\circ} 23^{\prime} 90^{\prime \prime} \mathrm{O}$ & 2850 \\
\hline \multirow[t]{2}{*}{6} & Santa Ana Tlacotenco, Milpa Alta, D.F. & SAT & $19^{\circ} 06^{\prime} 26^{\prime \prime} \mathrm{N}$ & \\
\hline & & & $99^{\circ} 00^{\prime} 11^{\prime \prime} \mathrm{O}$ & 3750 \\
\hline \multirow[t]{2}{*}{7} & La Soledad, San Antonio Calpulalpan, & LAS & $19^{\circ} 32^{\prime} 14^{\prime \prime} \mathrm{N}$ & \\
\hline & Tlaxcala & & $98^{\circ} 35^{\prime} 50^{\prime \prime} \mathrm{O}$ & 2440 \\
\hline \multirow[t]{2}{*}{8} & Milpa Alta, D. F. & MIA & $19^{\circ} 29^{\prime} 00^{\prime \prime} \mathrm{N}$ & \\
\hline & & & $99^{\circ} 16^{\prime} 00^{\prime \prime} \mathrm{O}$ & 2500 \\
\hline
\end{tabular}

de semilla y los lotes 5 al 8 fueron facilitados por la Comisión de Recursos Naturales (CORENA), de la Secretaría del Medio Ambiente del Gobierno del Distrito Federal.

\section{Siembra}

Para la producción de la planta se utilizó el procedimiento tradicional que se utiliza en el Vivero San Luis Tlaxiatemalco de la Comisión de Recursos Naturales de la Secretaria del Medio Ambiente del Gobierno del Distrito Federal (CORENA), con el fin de no alterar la posible aplicación de los resultados de este proyecto por parte de esta dependencia operativa, la cual es la encargada de la producción y abastecimiento de plantas para los programas de reforestación que se llevan a cabo en el Distrito Federal y áreas forestales aledañas.

Antes de la siembra se benefició la semilla mediante su desalado, separación por tamaños con ayuda de cribas y limpieza en una sopladora que separó las semillas viables de la basura y semillas vanas. Asimismo las semillas pasaron por un proceso de desinfección con cloro al $0,6 \%$, seguido de una imbibición por un periodo de 24 hrs y se eliminó el exceso de agua para posteriormente colocarlas en bolsas de plástico con fungicida Thiram horas antes de sembrarlas. 
La siembra de las ocho procedencias de $A$. religiosa se realizó en almácigo, el 28 y 29 de junio del 2004 y la germinación se presentó dentro de las 3 a 4 semanas posteriores a la misma (30 de julio al 3 de agosto del 2004). Las plántulas se trasplantaron a charolas de plástico rígido con 40 cavidades de $93 \mathrm{~cm}^{3}$ cada una, que contenían un sustrato de peat moss, agrolita y vermiculita en una proporción de 50:20:30 respectivamente y fertilizante Multicote (3,5 kg/m 3 de sustrato), el cual se aplicó posteriormente diluido en agua de riego cada ocho meses en promedio. De igual manera se les aplicó Micromax, con el fin de proporcionar a las plántulas los micronutrientes básicos. Finalmente, las charolas se llevaron al invernadero del vivero, donde se regaron por aspersión periódica (Fig. 3).

\section{Diseño experimental}

Las plántulas se mantuvieron en el invernadero, tiempo en el cual se llevó a cabo la evaluación de su crecimiento con base en la medición del diámetro en la base del tallo y la altura. La primera de estas variables se midió con un vernier digital (Mitutoyo, modelo No. CD-6"CS) y la altura con una regla métrica hasta la yema apical. Las mediciones se realizaron cada tres meses (29/09/2004, 10/01/2005, $6 / 04 / 2005$ y $28 / 07 / 2005)$.

Se usó un diseño experimental de bloques al azar con 8 bloques y 64 repeticiones por procedencia; en el cual cada charola fue considerada como un bloque y en cada una se muestrearon las ocho plántulas centrales a lo largo de la charola.

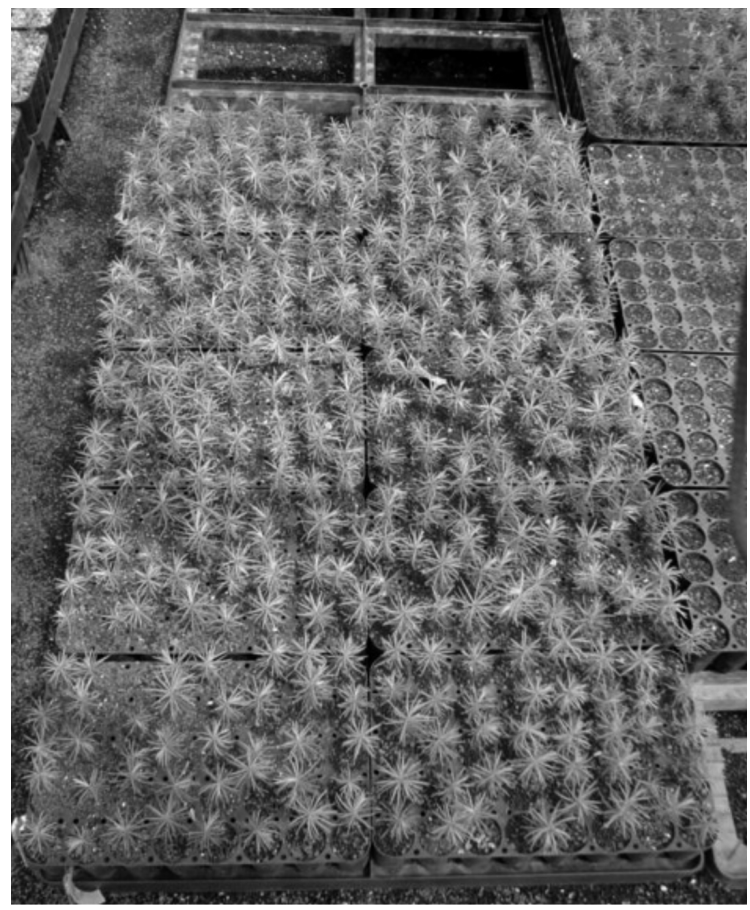

Figura 3. Plántulas de Abies religiosa en el vivero de San Luis Tlaxiatemalco de la CORENA. 


\section{Análisis estadístico}

Se obtuvo el promedio de la altura y diámetro basal para cada procedencia en cada uno de los bloques por cada fecha de medición y, con el fin de obtener la tasa de crecimiento, se obtuvo el logaritmo de dichos promedios para realizar un ajuste de mínimos cuadrados entre las cuatro fechas de evaluación. Una vez determinada la tasa de crecimiento del diámetro basal y la altura de cada procedencia, se realizó un análisis de varianza $(\alpha=0,05)$ y por último se llevó a cabo una comparación post- hoc o a posteriori, para saber en concreto cual o cuales eran las procedencias que presentaban diferencias en su respuesta entre sí mediante una prueba de Tukey.

\section{RESULTADOS}

\section{Altura}

Los valores promedio de altura por cada fecha de medición y para cada proce- dencia se muestran en la tabla 2. En la primera medición los valores fueron muy similares entre las procedencias; sin embargo hacia la segunda y tercera medición era ya posible observar ciertas diferencias entre ellas, lo cual resultó claramente notorio en los valores medios obtenidos en la última medición, en la cual el mayor valor se presentó en la procedencia de San Nicolás Coatepec y el menor desarrollo en Cofre de Perote (Tabla 2).

En la figura 4 se observa el desarrollo en altura y su ajuste de acuerdo con el procedimiento referido por cada fecha de medición. En el periodo comprendido de enero a abril del 2005 las procedencias mostraron una velocidad de crecimiento menor en comparación con los periodos de septiembre a enero y de abril a julio, en el cual se presentaron las mayores tasas de crecimiento. Este comportamiento es muy similar al que se esperaría encontrar en plántulas que se desarrollan bajo condiciones de campo,

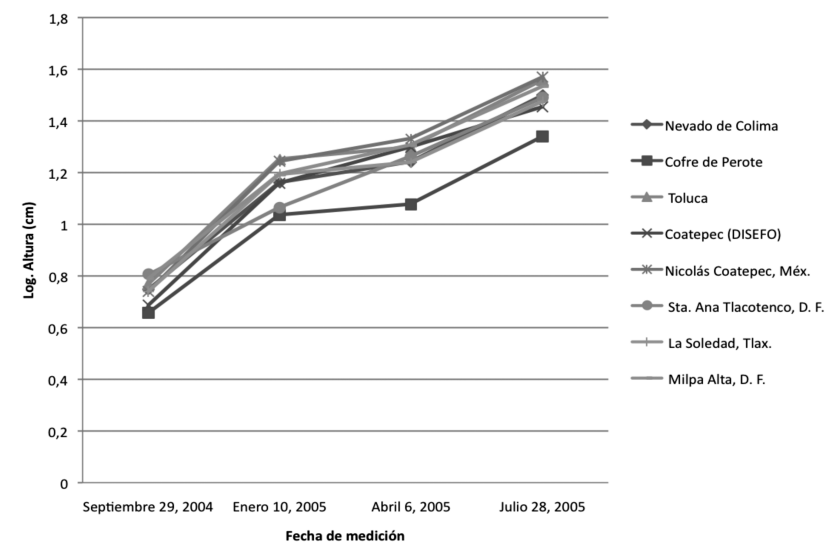

Figura 4. Tasas de crecimiento en altura de ocho procedencias de Abies religiosa cultivadas en vivero. 


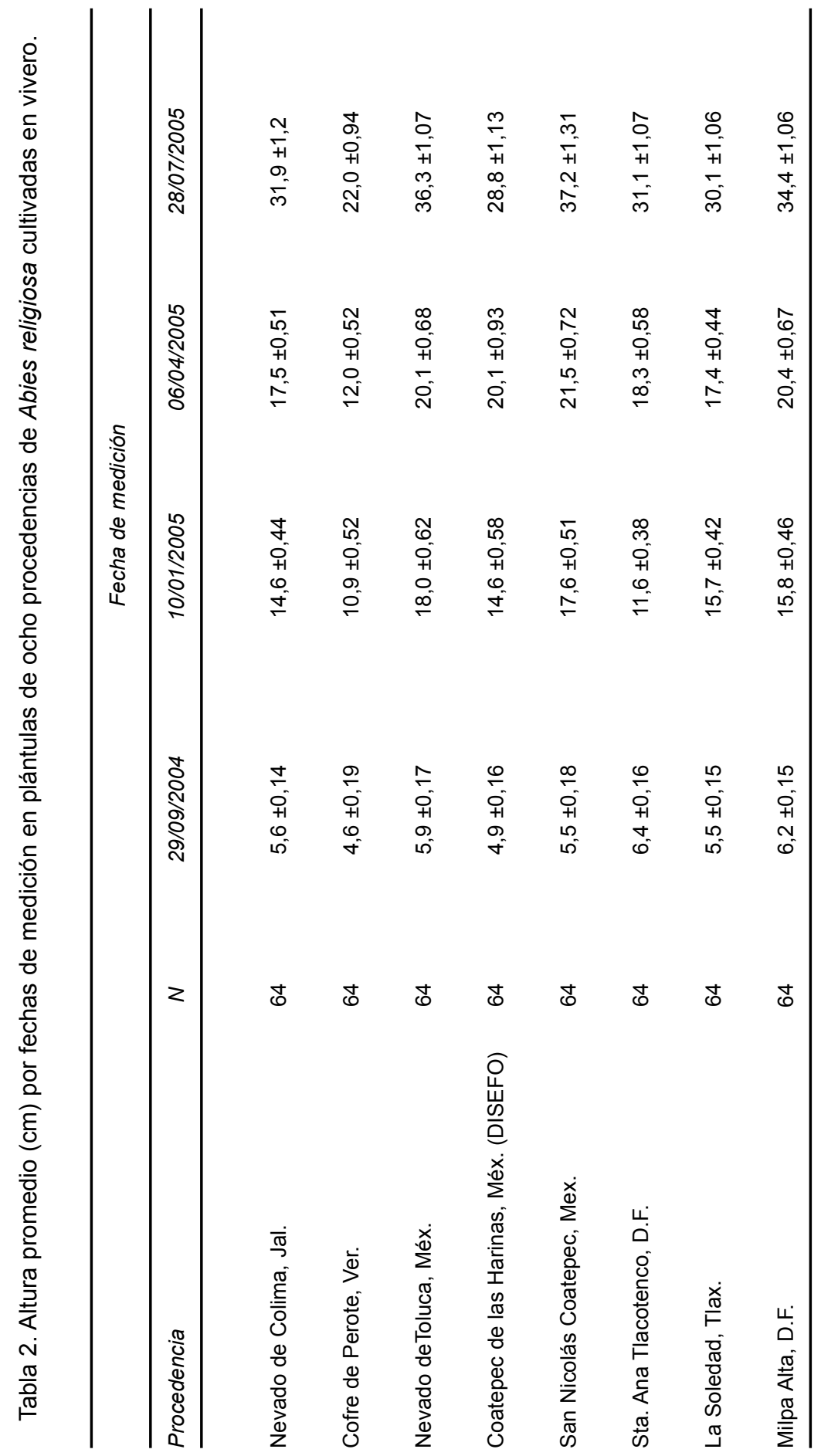


es decir, que crezcan más durante la temporada de mayor temperatura y lluvias y menos durante la temporada fría y de estiaje, lo que indica una respuesta fenológica muy asociada a las condiciones ambientales en esta especie, independiente de la cantidad de riego y que obedece más a cambios de temperatura y luz (fotoperiodo), relacionados con factores genéticos de control.

Los resultados del análisis de varianza practicado a los datos de altura indican que se registraron diferencias significativas $(\alpha=0,05)$ entre las ocho procedencias (Tabla 3 ).

Con base en lo anterior, se procedió a realizar una prueba de comparación de medias cuyos resultados se presentan en la tabla 4, en la cual se aprecia que la procedencia Cofre de Perote presentó una altura significativamente menor a la de las otras procedencias mientras que la de San Nicolás Coatepec resulto ser significativamente mayor a las de Santa Ana Tlacotenco, La Soledad, Coatepec (DISEFO) y Cofre de Perote (Tabla 4).

\section{Tasas de crecimiento}

Aunado al parámetro de altura, se realizó una prueba de análisis de varianza para comparar las tasas de crecimiento en altura entre las 8 procedencias de $A$. religiosa. Los resultados permitieron determinar que existían diferencias significa-

Tabla 3. Resultados del análisis de varianza a los valores de la altura en plántulas de ocho procedencias de Abies religiosa cultivadas en vivero.

\begin{tabular}{lccccc}
\hline FV & GI & SC & CM & $F$ & Sig Obs \\
\hline Total & 63 & 2002,515 & & & \\
Tratamientos & 7 & 1315,07988 & 187,868555 & 15,3041921 & 0,0000 \\
Error & 56 & 687,435117 & 12,2756271 & & \\
\hline
\end{tabular}

$\mathrm{FC}=63441,0156$

Tabla 4. Resultados de la prueba de Tukey en los valores medios de altura en plántulas de ocho procedencias de Abies religiosa cultivadas en vivero.

\begin{tabular}{lcc}
\hline Procedencia & Medias & $*$ \\
\hline San Nicolás Coatepec, Méx. & 37,2 & A \\
Nevado de Toluca, Méx. & 36,3 & AB \\
Milpa Alta, D.F. & 34,4 & ABC \\
Nevado de Colima, Jal. & 31,9 & ABCD \\
Sta. Ana Tlacotenco, D.F. & 31,1 & BCD \\
La Soledad, Tlax. & 30,1 & CD \\
Coatepec, Méx. (DISEFO) & 28,8 & D \\
Cofre de Perote, Ver. & 22,0 & E
\end{tabular}

* Los valores de media con diferente letra difieren significativamente entre sí. 
tivas $(\alpha=0,05)$ entre estas (Tabla 5$)$, por lo que se procedió a realizar una prueba de comparación de medias para dichos datos (Tabla 6).

Se registraron diferencias significativas entre las procedencias de San Nicolás Coatepec y Cofre de Perote, las cuales registraron el mayor y menor valor respectivamente. Tales resultados coinciden con los obtenidos en el análisis del promedio de la altura para estas dos procedencias. Asimismo, las procedencias de San Nicolás Coatepec, Coatepec (DISEFO) y Toluca, todas pertenecientes al Estado de México, se agrupan entre las tasas de crecimiento mayores.

\section{Diámetro}

En la tabla 7 se presentan los valores de diámetro promedio en cada fecha de medición, para cada procedencia bajo condiciones de vivero y, al igual que en la altura, se presentaron valores muy similares en la medición inicial, pero hacia el final del periodo de muestreo fue evidente la variación entre procedencias, pues en las plántulas provenientes de Coatepec (DISEFO) se registró el mayor crecimiento en diámetro, con un promedio de 4,29 $\mathrm{mm}$ y en la de Cofre de Perote se presentó la menor $(3,44 \mathrm{~mm})$.

Tabla 5. Resultados del análisis de varianza a los valores de la tasa de crecimiento en altura en plántulas de ocho procedencias de Abies religiosa cultivadas en vivero.

\begin{tabular}{lccccc}
\hline FV & GI & SC & CM & $F$ & Sig Obs \\
\hline Total & 63 & 0,0000039 & & & \\
Tratamientos & 7 & 0,0000011 & 0,00000017 & 3,44370341 & 0,0042 \\
Error & 56 & 0,0000027 & 0,000000049 & & \\
\hline
\end{tabular}

$\mathrm{FC}=0,000357666$

Tabla 6. Resultados de la prueba de Tukey en los valores medios de tasa de crecimiento en altura en plántulas de ocho procedencias de Abies religiosa cultivadas en vivero.

\begin{tabular}{lcc}
\hline Tratamiento & Media & * \\
\hline San Nicolás Coatepec & 0,0026 & $\mathrm{~A}$ \\
Coatepec, Méx. (DISEFO) & 0,0025 & $\mathrm{AB}$ \\
Nevado de Toluca, Mex. & 0,0024 & $\mathrm{AB}$ \\
Milpa Alta, D.F. & 0,0024 & $\mathrm{AB}$ \\
Nevado de Colima, Jal. & 0,0024 & $\mathrm{AB}$ \\
La Soledad, Tlax. & 0,0023 & $\mathrm{AB}$ \\
Sta. Ana Tlacotenco & 0,0023 & $\mathrm{AB}$ \\
Cofre de Perote, Ver. & 0,0021 & $\mathrm{~B}$ \\
\hline
\end{tabular}

*Las medias con diferente letra difieren significativamente entre sí. 


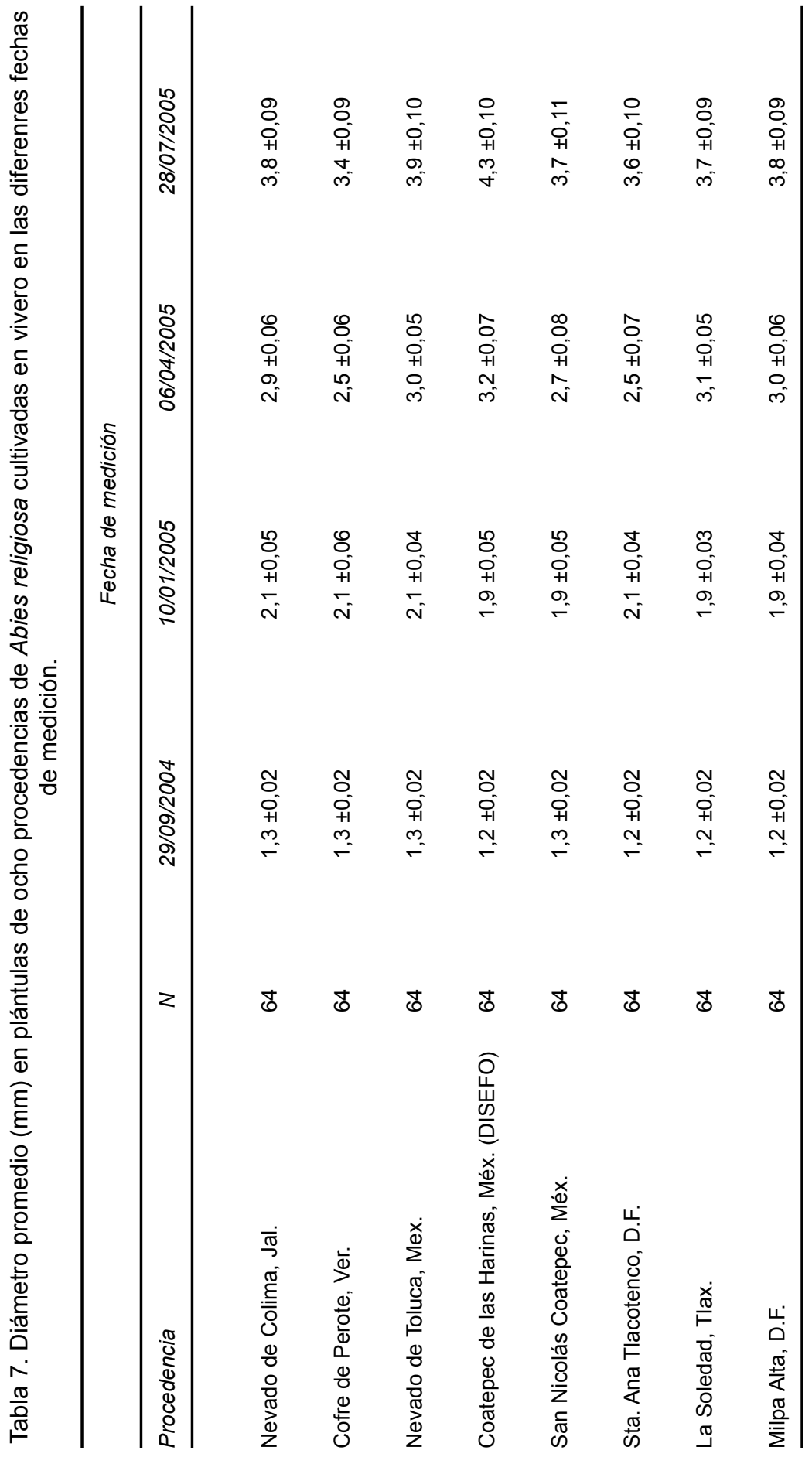


La figura 5 muestra que Las tasas de crecimiento registradas en esta variable fueron muy similares entre procedencias al principio del experimento, sin embargo se fueron diferenciando a partir de enero y las plántulas de Santa Ana Tlacotenco y Cofre de Perote presentaron una menor velocidad de crecimiento. A diferencia de la tasa de crecimiento en altura, la de diámetro presentó un patrón más constante a lo largo del tiempo.

Los resultados del análisis de varianza practicados a los datos de diámetro se presentan en la tabla 8 , los cuales indican que se registraron diferencias significativas $(\alpha=0,05)$ entre las ocho procedencias. Con base en lo anterior, se procedió a realizar una prueba de comparación de medias cuyos resultados se presentan en la tabla 9, en la cual se observa que las plántulas de la procedencia Cofre de Perote presentaron un diámetro significativamente menor a las de Nevado de Toluca y Coatepec (DISEFO). Cabe destacar que el diámetro de las plántulas de ésta última procedencia fue significativamente mayor al de todas las procedencias, excepto las de Nevado de Toluca.

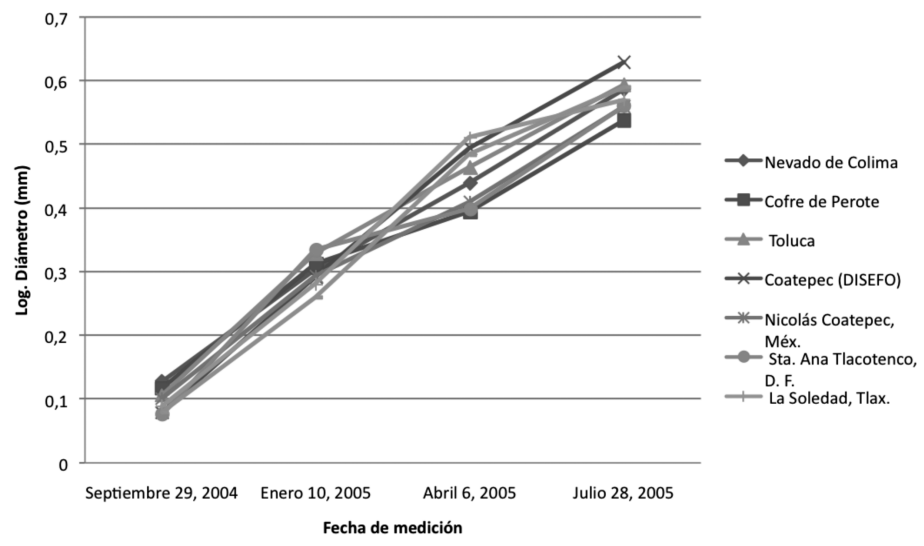

Figura 5. Tasa de crecimiento en diámetro de ocho procedencias de Abies religiosa de septiembre del 2004 a julio del 2005.

Tabla 8. Resultados del análisis de varianza a los valores de diámetro en plántulas de ocho procedencias de Abies religiosa cultivadas en vivero.

\begin{tabular}{lccccc}
\hline FV & GI & SC & CM & $F$ & Sig Obs \\
\hline Total & 63 & 8,59270134 & & & \\
Tratamientos & 7 & 3,50699646 & 0,50099949 & 5,51663384 & 0,0000734192 \\
Error & 56 & 5,08570488 & 0,09081616 & & \\
\hline
\end{tabular}

$F C=916,30127$ 
Tabla 9. Resultados de la prueba de Tukey en los valores medios de diámetro en plántulas de ocho procedencias de Abies religiosa cultivadas en vivero.

\begin{tabular}{lcc}
\hline Procedencia & Medias & $*$ \\
\hline Coatepec, Méx. (DISEFO) & 4,3 & A \\
Nevado de Toluca, Méx. & 3,9 & AB \\
Nevado de Colima, Jal. & 3,8 & BC \\
Milpa Alta, D. F. & 3,8 & BC \\
Nicolás Coatepec, Méx. & 3,7 & BC \\
La Soledad, Tlax. & 3,7 & BC \\
Sta. Ana Tlacotenco & 3,6 & BC \\
Cofre de Perote, Ver. & 3,4 & $\mathrm{C}$ \\
\hline
\end{tabular}

*Las medias con diferente letra difieren significativamente entre si.

Los resultados del análisis de varianza efectuado a los valores de tasas de crecimiento en diámetro en las 8 procedencias, indican que se presentaron diferencias significativas $(\alpha=0,05)$ entre éstas (Tabla 10) y, con base en este resultado, al realizar la prueba de comparación de medias entre procedencias se registró que al igual que el valor promedio de diámetro final, la tasa de crecimiento de la procedencia Coatepec (DISEFO) fue significativamente mayor al de las otras procedencias y la de Cofre de Perote fue la menor, difiriendo con Coatepec (DISEFO), Milpa Alta, D.F., La Soledad, Tlax. y Nevado de Toluca (Tabla 11).
Finalmente, en la tabla 12 se muestran los valores promedio finales de altura y diámetro que fueron alcanzados por las plántulas por procedencia, así como los de sus tasas de crecimiento en dichas variables. Se observa que en el caso de aquellas procedencias con un mayor tamaño final se registraron, como sería de esperar, los más altos valores de tasas de crecimiento. No obstante lo anterior, la tasa de crecimiento es una medida más certera para determinar qué procedencia creció más en grosor, particularmente cuando la medida inicial de la evaluación no es similar por tener dimensiones diferentes.

Tabla 10. Resultados del análisis de varianza a los valores de la tasa de crecimiento en diámetro en plántulas de ocho procedencias de Abies religiosa cultivadas en vivero.

\begin{tabular}{lccccc}
\hline FV & GI & $S C$ & $C M$ & $F$ & Sig Obs \\
\hline Total & 63 & 0,000002 & & & \\
Tratamientos & 7 & 0,0000012 & 0,00000017 & 12,5872159 & 0,0000 \\
Error & 56 & 0,0000008 & 0,000000014 & & \\
\hline
\end{tabular}

$\mathrm{FC}=0,0001629$ 
Tabla 11. Resultados de la prueba de Tukey en los valores medios de tasa de crecimiento en diámetro en plántulas de ocho procedencias de Abies religiosa cultivadas en vivero.

\begin{tabular}{lll}
\hline Procedencia & Media & $*$ \\
\hline Coatepec, Méx. (DISEFO) & 0,0019 & A \\
Milpa Alta, D.F. & 0,0017 & B \\
La Soledad, Tlax. & 0,0017 & B \\
Nevado de Toluca, Méx. & 0,0016 & B \\
Nevado de Colima, Jal. & 0,0015 & BC \\
Sta. Ana Tlacotenco & 0,0015 & BC \\
Nicolás Coatepec, Méx. & 0,0015 & BC \\
Cofre de Perote, Ver. & 0,0014 & C \\
\hline
\end{tabular}

*Las medias con diferente letra difieren significativamente entre sí.

Tabla 12. Valores promedio en altura, diámetro y sus respectivas tasas de crecimiento en plántulas de ocho procedencias de Abies religiosa cultivadas en vivero.

\begin{tabular}{|c|c|c|c|c|c|c|c|c|}
\hline Procedencia & Altura & $\begin{array}{c}\text { Nivel } \\
\text { de } \\
\text { signif. }\end{array}$ & $\begin{array}{c}\text { Tasa } \\
\text { de } \\
\text { crec. }\end{array}$ & $\begin{array}{l}\text { Nivel } \\
\text { de } \\
\text { signif. }\end{array}$ & Diámetro & $\begin{array}{l}\text { Nivel } \\
\text { de } \\
\text { signif. }\end{array}$ & $\begin{array}{c}\text { Tasa } \\
\text { de crec. } \\
\text { diámetro }\end{array}$ & $\begin{array}{c}\text { Nivel } \\
\text { de } \\
\text { signif. }\end{array}$ \\
\hline San Nicolás Coatepec, Méx. & 37,23 & A & 0,003 & A & 3,72 & $\mathrm{BC}$ & 0,0015 & $\mathrm{BC}$ \\
\hline Nevado de Toluca, Méx. & 36,28 & $A B$ & 0,002 & $A B$ & 3,94 & $A B$ & 0,0016 & B \\
\hline Milpa Alta, D. F. & 34,43 & $\mathrm{ABC}$ & 0,002 & $A B$ & 3,77 & $\mathrm{BC}$ & 0,0017 & B \\
\hline Nevado de Colima, Jal. & 31,94 & $A B C D$ & 0,002 & $A B$ & 3,78 & $\mathrm{BC}$ & 0,0015 & $\mathrm{BC}$ \\
\hline Sta. Ana Tlacotenco, D. F. & 31,12 & $\mathrm{BCD}$ & 0,002 & $A B$ & 3,64 & $\mathrm{BC}$ & 0,0015 & $\mathrm{BC}$ \\
\hline La Soledad, Tlax. & 30,08 & CD & 0,002 & $A B$ & 3,69 & $\mathrm{BC}$ & 0,0017 & B \\
\hline Coatepec, Méx. (DISEFO) & 28,75 & $\mathrm{D}$ & 0,003 & $A B$ & 4,29 & A & 0,0019 & A \\
\hline Cofre de Perote, Ver. & 22,01 & $E$ & 0,002 & $B$ & 3,44 & $\mathrm{C}$ & 0,0014 & $\mathrm{C}$ \\
\hline
\end{tabular}

*Los valores promedio con diferente letra difieren significativamente entre sí. 


\section{DISCUSIÓN}

En relación con el crecimiento en altura total, se encontraron diferencias significativas entre procedencias, en las cuales el menor valor se registró en Cofre de Perote y el inmediato, pero significativamente diferente, fue en las plántulas provenientes de Coatepec, Edo. de México. Asimismo, en la tasa de crecimiento sólo se distinguen dos grupos significativamente diferentes, de los cuales Cofre de Perote mostró el menor valor de ellos. Un patrón similar se registró en el tamaño final del diámetro y de su tasa de crecimiento, aunque en este caso la distinción de los grupos que se conforman a raíz de las diferencias registradas es menos uniforme.

Si bien el tiempo de evaluación en vivero fue relativamente pequeño, los datos derivados de este trabajo permitieron distinguir una respuesta claramente diferente en la altura de las plántulas de la procedencia Cofre de Perote, que se manifestó asimismo en los valores de diámetro basal en el cual fue diferente por lo menos con dos procedencias. Con base en lo anterior, los resultados no permiten determinar una posible variación que esté relacionada con un factor altitudinal o latitudinal de las procedencias.

Una reducida variación entre poblaciones de $A$. religiosa fue reportada por Aguirre-Planter et al. (2000), quienes refieren que en las coníferas es común encontrar una gran diversidad interna en las poblaciones, pero baja entre éstas, debido en parte a un extensivo flujo génico y la longevidad de los individuos. Una respuesta similar es la que reportan Juárez-Agis et al. (2006) para Pseudotsuga menziesii (Mirb.) Franco, quienes no encontraron diferencias en el tamaño de plántulas de 9 meses de edad de diferentes poblaciones.
Con base en lo anterior, es posible considerar que la variación registrada en las plántulas de Cofre de Perote se relacione más con factores climáticos que influyen en esas poblaciones y que determinan un inicio y un detenimiento del crecimiento anticipado y diferente de la población con respecto a las demás procedencias. Lamentablemente en este trabajo no se registró la fenología de las poblaciones bajo estudio, lo que habría ayudado a dilucidar este supuesto. No obstante lo anterior, la respuesta fenológica entre procedencias está bien documentada, especialmente en aquellas especies con amplios rangos latitudinales de distribución, como sería el caso de Liquidambar stiracyflua L.

\section{CONCLUSIONES}

No obstante las diferencias significativas registradas en el crecimiento en altura y diámetro en plántulas de diferentes procedencias de Abies religiosa, no se encontró un patrón de variación latitudinal, longitudinal o altitudinal entre las mismas.

\section{RECONOCIMIENTOS}

Este trabajo forma parte del proyecto intitulado "Introducción de procedencias para la restauración de los bosques en declinación del Distrito Federal. Etapa 1: Pinus hartwegii y Abies religiosa, el cual fue posible realizarlo en forma parcial gracias al apoyo económico del Fondo Sectorial Conafor-Conacyt. Asimismo, el apoyo del Vivero San Luis Tlaxiatemalco fue fundamental para la producción y mantenimiento de la planta y merece nuestro reconocimiento la desinteresada colaboración del Ing. Salvador Castro Zavala. De igual manera, la realización de este trabajo hubiera sido difícil si no se hubiera contado con la ayuda de los 
alumnos de la Carrera de Biología de la Facultad de Ciencias, que colaboraron en el taller relacionado con este trabajo.

\section{REFERENCIAS}

Aguirre-Planter, E., G.R. Furnier y L.E. Eguiarte. 2000. Low levels of genetic variation within and high levels of genetic differentiation among populations of species of Abies from southern Mexico and Guatemala. American Journal of Botany 87(3):362-371.

Alvarado, R.D., L.I. de Bauer y A.J. Galindo. 1992. Decline of sacred fir (Abies religiosa) in a forest Park south of Mexico City. Environmental Pollution 80: 115-121.

Alvarado-Rosales, D, y T. HernándezTejeda. 2002. Decline of sacred fir in the Desierto de los Leones National Park. In: Urban air pollution and forests: resources at risk in the Mexico City air basin. Fenn M., L.I. Bauer y T. Hernández, eds. Springer-Verlag, Nueva York.

Ashby, W.C. 1987. Forests. In: W.R. Jordan, M.E. Gilpin y J.D. Aber, eds. Restoration ecology: a synthetic approach to ecological research. Cambridge University Press, Cambridge, Inglaterra. p:89-108.

Benavides-Meza, H.M., B. Ortega R., M. de la P. Medina B. y P. de la Garza L. 1994. Notas del Curso de Dasonomía Urbana. Centro Nacional de Investigación Disciplinaria en Conservación y Mejoramiento de Ecosistemas Forestales, Instituto Nacional de Investigaciones Forestales y Agropecuarias. sp.

Burley, J. 1969. Metodología de los ensayos de procedencia de especies forestales. Unasylva 23(3):24-28.
Calderón de Rzedowski, G. y J. Rzedowski. 2001. Flora fanerogámica del Valle de México. 2a Edición. Instituto de Ecología A. C. y Comisión Nacional para el Conocimiento y Uso de la Biodiversidad. Pátzcuaro, Michoacán, México. $1406 \mathrm{p}$.

Cibrián, T.D. 1989. Air pollution and forest decline near Mexico City. Environmental Monitoring and Assessment 12:49-58.

Cielsa, W.M. y S.J. Macías. 1987. Desierto de los Leones. A forest in crisis. American Forest. November/December.

Climent, J., R. Chambel, D. Barba, J. Voltas y R. Alía. 2008. Evaluación genética de la planta forestal: conceptos y resultados disponibles para rodales de pinos españoles. Boletín del CIDEU 67:69-82.

Comisión Nacional de Áreas Naturales Protegidas (CONANP). 2006. Programa de Conservación y Manejo Parque Nacional "Desierto de los Leones". México. 172 p. Disponible en: http://www.conanp.gob.mx/pdf_progra ma_manejo/Desierto_ok.pdf. (21 de abril de 2009).

Cortés-Barrera, E.N., C. Nieto de PascualPola, y M.A. Pérez-Hernández. 2005. Regeneración natural de Abies religiosa (H.B.K.) Schl et Cham. en dos localidades del Distrito Federal. In: Memorias del VII Congreso Mexicano de Recursos Forestales. 26-28 de octubre de 2007. Universidad Autónoma de Chihuahua. p:202-203.

García-Quintana Y., A. Álvarez-Brito y E. Guizar-Nolazco. 2007. Ensayo de procedencias de Pinus caribea var. caribea en alturas de pizarras, Viñales, Pinar del Río, Cuba. Revista Chapingo: Serie Ciencias Forestales y del Ambiente 13(2):125-129. 
Gómez-González, R. 2003. Estado del conocimiento de Abies religiosa (H.B.K.) Schl et Cham. Tesis de Licenciatura, Universidad Autónoma Chapingo. 450 p.

González-Medina, R.E., M. Mendoza B. y D. Alvarado-Rosales. 2010. Exposición a ozono en relación a vitalidad en un bosque de oyamel (Abies religiosa (Kunth) Schltdl. \& Cham). Madera y Bosques 16(4):7-19

Granados-Sánchez, D. y G.F. López-Ríos. 2001. Declinación Forestal. Revista Chapingo: Serie Ciencias Forestales y del Ambiente 7(1):5-13.

Juárez-Agis, A., J. López-Upton, J.J. VargasHernández y C. Sáenz Romero. 2006. Variación geográfica en la germinación y crecimiento inicial de plántulas de Pseudotsuga mensiezii de México. Agrociencia 40 (6):783-792.

Madrigal-Sánchez, X. 1967. Contribución al conocimiento de la ecología de los bosques de oyamel (Abies religiosa $(\mathrm{H}$. B. K.) Schl. Et Cham.) en el Valle de México. Boletín Técnico No. 18. INIF. México, D. F. 94 p.

Manzanilla-Bolio, H. 1974. Investigaciones epidométricas y silvícolas en bosques mexicanos de Abies religiosa. Dirección General de Información y Relaciones Públicas, Secretaría de Agricultura y Ganadería. México, D.F. 165 p.

Martínez, M. 1948. Los Abies mexicanos. In: Anales del Instituto de Biología de México. No. 1, Tomo XIX. Universidad Nacional Autónoma de México. México. 104 p.

Martínez, M. 1953. Las Pináceas Mexicanas. Secretaría de Agricultura y Ganadería, Secretaría de Recursos Forestales y de Caza. México. 362 p.
Moreno Ch., L.R., M.A. López L., E. Estañol B. y A. Velázquez M.. 2002. Diagnóstico de necesidades de fertilización de Abies religiosa (H.B.K.) Schl. et Cham. en vivero mediante el DRIS. Madera y Bosques 8(1):51-60.

Nieto de Pascual-Pola, C. 1995. Estudio sinecológico del bosque de oyamel de la Cañada Contreras, D.F. Rev. Cien. For. en Mex. 20 (77):3-34.

Nieto de Pascual-Pola, C. 2004. Regeneración del oyamel, Abies religiosa (H.B.K.) Schl et Cham., bajo condiciones naturales y controladas. Tesis de Doctorado, Facultad de Ciencias, UNAM. $142 \mathrm{p}$.

Nieto de Pascual-Pola, C., M.A. Musálem Santiago y J. Ortega Alcalá. 2003. Estudio de algunas características de conos y semillas de $A$. religiosa (H.B.K.) Schl. et Cham. Agrociencia 37(5):521-531.

FAO (Organización de las Naciones Unidas para la Agricultura y la Alimentacion). 1971. Informe de la segunda reunión del cuadro de expertos de la FAO en recursos genéticos forestales. Georgia, EUA.

Pérez-Suárez, M., V.M. Cetina-Alcalá, A. Aldrete, M.E. Fenn y L.L LandoisPalencia. 2006. Química de la precipitación pluvial en dos bosques de la cuenca de la Ciudad de México. Agrociencia 40(2):239-248.

Rodríguez-Trejo, D.A. 2006. Notas sobre el diseño de plantaciones de restauración. Revista Chapingo: Serie Ciencias Forestales y del Ambiente 12(2):111123.

Saavedra-Romero, L. de L., D. AlvaradoRosales, J. Vargas-Hernández y $\mathrm{T}$. Hernández-Tejeda. 2003. Análisis de la 
precipitación pluvial en bosques de Abies religiosa (HBK.) Schltdl. et Cham., en el sur de la Ciudad de México. Agrociencia 37 (1):57-63.

Santiago-Trinidad, O. 2002. Evaluacion del crecimiento en vivero de plantulas de cinco especies de coniferas producidas en tres mezclas de sustratos y tres tamaños de contenedor. Tesis de Maestría, División de Ciencias Forestales, Universidad Autónoma Chapingo. Chapingo, México. 241p.

Vázquez-Soto, J. 1987. El saneamiento y la limpia forestal en el Desierto de los Leones. Comisión Coordinadora para el Desarrollo Rural (COCODER). México, D.F. 24 p.
Vázquez-Soto, J. 1988. Los tratamientos silvícolas del Desierto de los Leones sus fundamentos. Comisión Coordinadora para el Desarrollo Rural (COCODER). Departamento del Distrito Federal. México, D.F. 34 p.

Weaver M.-J. y R.-J. Stipes. 1988. White pine decline: a case study from Virginia landscapes. Journal of Arboriculture 14(5):109-120.

Zobel, B.J. y J.T. Talbert. 1984. Applied forest tree improvement. John Wiley and Sons. Nueva York, EUA. 505 p. 